\title{
Imaging in animal models
}

\author{
Eika Webb ${ }^{1}$, Ming Yuan ${ }^{1,2 *}$, Nicholas R Lemoine ${ }^{1,2}$ and Yaohe Wang ${ }^{1,2 *}$ \\ ${ }^{1}$ Center for Molecular Oncology, Barts Cancer Institute, Queen Mary University of London, London, UK \\ ${ }^{2}$ Sino-British Research Centre for Molecular Oncology, National Center for International Research in Cell and Gene Therapy, Zhengzhou University, Zhengzhou, \\ China
}

\begin{abstract}
Advances in imaging techniques have significantly improved the choices available for in vivo imaging of animal research, particularly in the field of oncology. Development of existing technology has led to major advances in the miniaturisation of imaging techniques such as MRI, PET and CT enabling higher resolution imaging of smaller animals. One of the emerging growth areas is optical imaging, such as bioluminescence and fluorescence as a noninvasive technique to image orthotopic tumours models in mice. Recent developments in microscopic imaging techniques allow high resolution imaging of cancer cells in vivo in animal models. Another growth area is the development of multimodal scanners such as CT-PET to overcome individual limitations of a modality and to provide greater detail of tumours. This review discusses the strengths and limitations of established methods as well as more recent developments in preclinical imaging.
\end{abstract}

\section{Introduction}

Imaging has become an indispensable tool in oncological research. Recent developments in biomedical research have led to major advances in imaging techniques. Previously, scientific studies had been confined to examination of ex vivo animal tissues or cellular processes in vitro. However, with the development of new technology, a wide array of imaging techniques has become available for the use in small animal models (predominantly mice). Advances in this field have particular significance for oncological research for the detection, staging, prognosis and treatment of cancer in animal models of disease. Clinically established macroscopic modalities such as computerised tomography (CT), magnetic resonance imaging (MRI) and position emission tomography (PET) are useful modalities in the macroscopic imaging of small animal models.

Further, with the advent of orthotopic tumour models there is an emphasis on developing non-invasive techniques to image and monitor tumours that grow deep within the body cavity of the animal using optical imaging techniques [1]. Advances in optical imaging including bioluminescence and high resolution fluorescent imaging are paving the way towards more rapid, accurate and efficient methods of cancer imaging.

\section{Macroscopic imaging techniques}

Due to the technological difficulties involved in miniaturisation, CT, MRI, and PET were first developed for use in humans and later adapted for small animal models [2]. Specialised micro-CT machines rely on the differential absorption of tissues to $\mathrm{x}$-rays to produce high spatial resolution images of mice. In particular, micro-CT is used to detect bone metastases [3] and lung tumours in mice [4,5] due to the favourable contrast between soft tissue and bone [6,7]. Namati et al. describes the use of micro-CT on mouse models of lung cancer to track individual tumour growth rates from an early stage with high resolution. In another study [8], demonstrates the use of micro-CT to assess the efficacy of chemotherapy and provide non-invasive realtime imaging of the tumour burden. These studies indicate that micro-
CT is paving the way towards improved image guided evaluation of tumours in experimental models as well their response to therapeutic interventions. Unfortunately, one of the drawbacks of micro-CT is the use of iodinated contrast agents to achieve higher spatial resolutions, limiting repeated scanning of the same animal due to the administration of damaging doses of radiation [4].

In contrast to micro-CT and PET, micro-MRI utilises a magnetic field to generate images of the subject based on the varying resonance of different tissue types [9]. Therefore, micro-MRI enables repeated scans on the same animal as it does not expose them to ionising radiation. Similar to CT, micro- MRI has a high spatial resolution and has wide applications of use including functional, anatomical and molecular imaging [10]. The advantage of micro-MRI is that it provides excellent soft tissue resolution between $100-200 \mu \mathrm{m}$ [11] and even up to $50 \mu \mathrm{m}$ [12] in high strength magnetic fields, allowing imaging of tumour growth and development. Another strength of MRI is its sensitivity to biological processes including perfusion and flow, enabling imaging of tumour vasculature. Further, the use of dynamic contrast enhanced (DCE) MRI can be used to image markers of angiogenesis [13] The imaging of angiogenesis is an important area of cancer research as it provides insight into the microenvironment required for metastasis and aids the development of anti-angiogenic drugs [14,15]. However, the drawback of both CT and MRI is that they lack molecular specificity in contrast to PET which provides excellent specificity in tumour imaging [16].

PET uses systemically administered radiolabelled tracers such as 18F-FDG (fludeoxyglucose) to image biological processes and is a key clinical tool for the staging and assessment of tumour response to

Correspondence to: Yaohe Wang and Ming Yuan, Center for Molecular Oncology, Barts Cancer Institute, Queen Mary University of London, London, UK, E-mail: Yaohe.wang@qmul.ac.uk,m.yuan@qmul.ac.uk

Key words: cancer, imaging techniques, MRI, PET, CT

Received: March 18 2016; Accepted: April 05, 2016; Published: April 08, 2016 
treatment. [17]. Previously, PET was limited to image larger animal models [18] but with improvements in technology and imaging quality, it is increasingly being used on smaller animals. The strengths of PET include the almost unlimited depth of imaging and excellent molecular sensitivity [16]. Typical tracer doses are between 0.5 to $2 \mathrm{mCi}$ range [19] for rat studies however are limited by the mass and volume of tracer as well as the count rate ability of the scanner rather than the dose of radiation [20]. A further advantage of PET is that each animal can act as their own control which reduces variability in therapeutic trials [19].

However, one limitation of micro-PET is its lower spatial resolution, due to the difficulties involved in adapting human resolution $\sim 10 \mathrm{~mm}$ to $<1 \mathrm{~mm}$ in animal models [21]. Due to these disadvantages, there is increasing emphasis on multimodal imaging such as micro-CT/MRI although this impractical for many laboratories due to cost and need for specialist equipment. However, newer generation PET scanners are in development with higher resolution of around $1 \mu \mathrm{l}$ [11]. Further advances are expected in this field with refinement of the technology, to improve imaging of tumour cell response to therapeutic agents.

\section{Optical imaging}

Optical imaging technologies have evolved due to improvements in light penetration in tissues, to become important tools as they provide qualitative information in whole animal imaging of cancer biology. Optical imaging can be divided into bioluminescence and fluorescence. Optical imaging is based on the interaction of photons with tissue components and this interaction is characterized by three main criteria; light absorption, light scattering, and fluorescent emission [22].

Bioluminescence is a non-invasive method of monitoring of tumour growth in animal models. It involves using an exogenously administered substrate such as luciferase or a Renilla-based reporter to produce a light signal which is collected by a charge coupled device (CCD) camera [23]. One of the advantages of bioluminescence in animal studies is that there is very little background noise [24].

Similarly, fluorescence is an inexpensive and efficient method of optical imaging. This technique applies one wavelength of light to illuminate the sample, resulting in a shifted wavelength that is collected by a CCD camera [23]. This enables fluorescently labelled cancer cells to be tracked longitudinally allowing for the study of cell migration and metastasis [25]. However disadvantages exist, including the requirement of restraining and anaesthetising the animal as well as the injection of luciferin substrate.

Optical imaging is efficient and relatively cost-effective in comparison to other modalities however, it does suffer from weaknesses such as inferior spatial resolution, which limits its usage to molecular purposes and not anatomical imaging. Near infrared fluorescence imaging is being developed in order to overcome these limitations by focusing on the detection of exogenously administered contrast agents that emit fluorescence between 700-900 nm [26]. Recent animal studies have shown promising uses of near-infrared fluorescence imaging for the demarcation of subcutaneous and orthotopic xenograph tumours during surgery [27-29]. Resection of the tumours was carried out under guidance of near-infrared fluorescence imaging, enabling identification of any remaining cancerous cells in situ [30]. In addition, further studies have utilised optical imaging to detect cervical node metastases in orthotopic mouse models as all nodes that showed a fluorescent signal were found to be malignant metastases from squamous cell carcinoma $[31,32]$. Thus, near-infrared fluorescence imaging can be used to delineate tumour margins and any malignant cells remaining post resection, making it an invaluable tool for animal model imaging.

In the future, fluorescence models may increasingly be utilised in the diagnosis and monitoring of tumours due to its ability to provide real time imaging during surgery and in monitoring the effect of potential therapeutic agents in cancer treatment [33].

\section{Microscopic imaging techniques}

Recent developments in microscopic imaging techniques have enabled the transition from cellular imaging to animal models. Confocal microscopy is one such technique which utilises a special filter to eliminate out-of-focus fluorescent light, enabling the production of high quality micrographs with increased resolution and contrast. Due to its relative ease of use and its cost-effectiveness, confocal microscopy has become widespread. The drawback is that penetration depths are usually $<40 \mu \mathrm{m}$ and prolonged observation times can cause cell death due to phototoxicity [34].

However, a different technique, multiphoton microscopy (MPM) bypasses these limitations and has the ability to produce high resolution imaging of cancer cells in vivo in animal models [35]. MPM is increasingly being used to image intravital structures as it can reach resolutions of up to $500 \mu \mathrm{m}$ for extended periods of time (>72h) [36]. The ability of MPM to image deep into solid tumours enables tumour progression to be followed on a single cell level and promotes research into understanding tumour cell behaviour and metastasis. The ability of MPM to produce images in multiple colours allows for a greater understanding of the tumour microenvironment as it images tumour and stromal cells [37].

Recent developments in fibre-optic technology have been modified for use in intravital microscopy, furthering the possibility of imaging at orthotopic tumour locations. Small scale experimental systems are being adapted for use in rodent models and for clinical imaging [38-40]. Furthermore, intravital imaging has identified differences in tumour cell movement in animal models in comparison to in vitro models which could provide more information on tumour microenvironment and mechanisms of invasion and metastasis $[41,42]$.

\section{Future perspectives}

Multimodal imaging is a promising new area of development. Imaging using multiple modalities are increasingly being investigated for future use in order to circumvent the limitations of individual modalities. Multimodal scanning encompasses several techniques, allowing the imaging of multiple modalities in a single machine without transferring the animal. Combining anatomical imaging techniques such as MRI or CT with molecular techniques such as PET provides more detailed information than a single modality [43].

Similarly, micro-MRI used in conjunction with micro-PET enables imaging down to the molecular level, greater precision in the monitoring of cancer progression as well as tumour cell response to therapy. The acquisition of simultaneous results from PET/MRI gives a greater understanding of spatial as well as temporal data from MRI as well as functional information from PET [44]. A variety of multimodal scanners including; PET-CT, PET-MRI are in development to improve the visualisation of tumours $[43,45,46]$. It is hoped that the recent advances in imaging techniques will provide a greater understanding of the molecular basis of cancer, providing further insight into signalling pathways responsible for tumour growth and metastasis [46]. 


\section{Acknowledgment}

This work is supported by the MRC (MR/M015696/1) and CRUK Centre Grant (C16420/A18066).

\section{References}

1. Hoffman RM (2005) Orthotopic metastatic (MetaMouse) models for discovery and development of novel chemotherapy. Methods Mol Med 111: 297-322. [Crossref]

2. Hutchins GD, Miller MA, Soon VC, Receveur T (2008) Small animal PET imaging. ILAR J 49: 54-65. [Crossref]

3. Winkelmann C, Figueroa S, Sieckman G, Rold T, Hoffman T (2012) Non-invasive MicroCT imaging characterization and in vivo targeting of $\mathrm{BB} 2$ receptor expression of a PC-3 bone metastasis model. Mol Imaging Biol 14: 667-675. [Crossref]

4. Paulus MJ, Gleason SS, Kennel SJ, Hunsicker PR, Johnson DK (2000) High resolution $\mathrm{X}$-ray computed tomography: an emerging tool for small animal cancer research. Neoplasia 2: 62-70. [Crossref]

5. Hori Y, Takasuka N, Mutoh M, Kitahashi T, Kojima S, et al. (2008) Periodic analysis of urethane-induced pulmonary tumors in living A/J mice by respiration-gated X-ray microcomputed tomography. Cancer Sci 99: 1774-1777. [Crossref]

6. Namati E, Thiesse J, Sieren JC, Ross A, Hoffman EA, et al. (2010) Longitudina assessment of lung cancer progression in the mouse using in vivo micro-CT imaging. Med Phys 37: 4793-4805. [Crossref]

7. Rudyanto R, Bastarrika G, de Biurrun G, Agorreta J, Montuenga L, et al. (2013) Individual nodule tracking in micro-CT images of a longitudinal lung cancer mouse model. Med Image Anal 17: 1095-1105. [Crossref]

8. Fushiki H, Kanoh-Azuma T, Katoh M, Kawabata K, Jiang J, et al. (2009) Quantification of mouse pulmonary cancer models by microcomputed tomography imaging. Cancer Sci 100: 1544-1549. [Crossref]

9. Imaizumi M, Yamamoto S, Kawakami M, Aoki M, Sugiyama E, et al. (2009) Simultaneous imaging of magnetic resonance imaging and positron emission tomography by means of MRI-compatible optic fiber-based PET: a validation study in ex vivo rat brain. Jap J Radiol 27: 252-256. [Crossref]

10. Garbow J, Wang M, Wang Y, Lubet R, You M (2008) Quantitative Monitoring of Adenocarcinoma Development in Rodents by Magnetic Resonance Imaging. Clin Cancer Res 14: 1363-1367. [Crossref]

11. de Kemp RA, Epstein FH, Catana C, Tsui BM, Ritman EL (2010) Small-animal molecular imaging methods. J Nucl Med 51: 18S-32S. [Crossref]

12. Czernin J, Weber WA, Herschman HR (2006) Molecular imaging in the development of cancer therapeutics. Annu Rev Med 57: 99-118. [Crossref]

13. O'Connor JP, Jackson A, Parker GJ, Jayson GC (2007) DCE-MRI biomarkers in the clinical evaluation of antiangiogenic and vascular disrupting agents. Br J Cancer 96: 189-195. [Crossref]

14. Zwick S, Strecker R, Kiselev V, Gall P, Huppert J, et al. (2009) Assessment of vascular remodeling under antiangiogenic therapy using DCE-MRI and vessel size imaging. $J$ Magn Reson Imaging 29: 1125-1133. [Crossref]

15. Ali M, Janic B, Babajani-Feremi A, Varma N, Iskander A, et al. (2010) Changes in Vascular Permeability and Expression of Different Angiogenic Factors Following AntiAngiogenic Treatment in Rat Glioma. PLoS ONE 5: e8727. [Crossref]

16. Oteo M, Morcillo M (2015) Small Animal PET as Non-Invasive Tool for Preclinical Imaging. Acta Phys Pol A 127: 1483-1486.

17. Ben-Haim S, Ell P (2009) 18F-FDG PET and PET/CT in the evaluation of cancer treatment response. J Nucl Med 50: 88-99. [Crossref]

18. Watanabe M, Uchida H, Okada H, Shimizu K, Satoh N, et al. (1992) A high resolution PET for animal studies. IEEE Trans Med Imaging 11: 577-580. [Crossref]

19. Chatziioannou A, Tai YC, Doshi N, Cherry SR (2001) Detector development for microPET II: a 1 microl resolution PET scanner for small animal imaging. Phys Med Biol 46: 2899-2910. [Crossref]

20. Cherry SR, Gambhir SS (2001) Use of positron emission tomography in anima research. ILAR J 42: 219-232. [Crossref]

21. Chatziioannou AF (2002) Molecular imaging of small animals with dedicated PET tomographs. Eur J Nucl Med Mol Imaging 29: 98-114. [Crossref]
22. Kovar JL, Simpson MA, Schutz-Geschwender A, Olive DM (2007) A systematic approach to the development of fluorescent contrast agents for optical imaging of mouse cancer models. Anal Biochem 367: 1-12. [Crossref]

23. Hoffman R (2012) Cellular and Subcellular Imaging in Live Mice Using Fluorescent Proteins. Curr Pharm Biotech 13: 537-544. [Crossref]

24. O'Neill K, Lyons SK, Gallagher WM, Curran KM, Byrne AT (2010) Bioluminescent imaging: a critical tool in pre-clinical oncology research. J Pathol 220: 317-327. [Crossref]

25. Bouvet M, Hoffman RM (2015) Tumor imaging technologies in mouse models. Methods Mol Biol 1267: 321-348. [Crossref]

26. Gioux S, Coutard JG, Berger M, Grateau H, Josserand V, et al. (2012) FluoSTIC: miniaturized fluorescence image-guided surgery system. J Biomed Opt 17: 106014. [Crossref]

27. Gleysteen JP, Newman JR, Chhieng D, Frost A, Zinn KR, et al. (2008) Fluorescent labeled anti-EGFR antibody for identification of regional and distant metastasis in a preclinical xenograft model. Head Neck 30: 782-789. [Crossref]

28. Keereweer S, Kerrebijn J, Mol I, Mieog J, Van Driel P, et al. (2011) Optical imaging of oral squamous cell carcinoma and cervical lymph node metastasis. Head Neck 34 1002-1008. [Crossref]

29. Day KE, Sweeny L, Kulbersh B, Zinn KR, Rosenthal EL (2013) Preclinical comparison of near-infrared-labeled cetuximab and panitumumab for optical imaging of head and neck squamous cell carcinoma. Mol Imaging Biol 15: 722-729. [Crossref]

30. Keramidas M, Josserand V, Righini C, Wenk C, Faure C, et al. (2010) Intraoperative near-infrared image-guided surgery for peritoneal carcinomatosis in a preclinical experimental model. Br J Surg 97: 737-743. [Crossref]

31. Heath CH, Deep NL, Sweeny L, Zinn KR, Rosenthal EL (2012) Use of panitumumabIRDye800 to image microscopic head and neck cancer in an orthotopic surgical model. Ann Surg Oncol 19: 3879-3887. [Crossref]

32. Rosenthal E, Kulbersh B, King T, Chaudhuri T, Zinn K (2007) Use of fluorescent labeled anti-epidermal growth factor receptor antibody to image head and neck squamous cell carcinoma xenografts. Mol Cancer Ther 6: 1230-1238. [Crossref]

33. Tanaka E, Choi H, Fujii H, Bawendi M, Frangioni J (2006) Image-Guided Oncologic Surgery Using Invisible Light: Completed Pre-Clinical Development for Sentinel Lymph Node Mapping. Ann Surg Oncol 13: 1671-1681. [Crossref]

34. Wang W, Wyckoff JB, Frohlich VC, Oleynikov Y, Huttelmaier S, et al. (2002) Single cell behavior in metastatic primary mammary tumors correlated with gene expression patterns revealed by molecular profiling. Cancer Res 62: 6278-6288. [Crossref]

35. Gatesman Ammer A, Hayes K, Martin K, Zhang L, Spirou G, et al. (2011) Multi-photon Imaging of Tumor Cell Invasion in an Orthotopic Mouse Model of Oral Squamous Cell Carcinoma. $J$ Vis Exp. [Crossref]

36. Leong HS, Steinmetz NF, Ablack A, Destito G, Zijlstra A, et al. (2010) Intravital imaging of embryonic and tumor neovasculature using viral nanoparticles. Nat Protoc 5: 1406-1417. [Crossref]

37. Wyckoff J, Wang W, Lin EY, Wang Y, Pixley F, et al. (2004) A paracrine loop between tumor cells and macrophages is required for tumor cell migration in mammary tumors. Cancer Res 64: 7022-7029. [Crossref]

38. Flusberg BA, Cocker ED, Piyawattanametha W, Jung JC, Cheung EL, et al. (2005) Fiber-optic fluorescence imaging. Nat Methods 2: 941-950. [Crossref]

39. Cho C, Ablack A, Leong H, Zijlstra A, Lewis J (2011) Evaluation of Nanoparticle Uptake in Tumors in Real Time Using Intravital Imaging. J Vis Exp. [Crossref]

40. Kiesslich R, Goetz M, Vieth M, Galle P, Neurath M (2007) Technology Insight: confocal laser endoscopy for in vivo diagnosis of colorectal cancer. Nat Clin Pract Oncol 4: 480-490. [Crossref]

41. Fukumura D, Duda D, Munn L, Jain R (2010) Tumor Microvasculature an Microenvironment: Novel Insights Through Intravital Imaging in Pre-Clinical Models. Microcirculation 17: 206-225. [Crossref]

42. van de Ven AL, Kim P, Ferrari M, Yun SH (2013) Real-time intravital microscopy of individual nanoparticle dynamics in liver and tumors of live mice. Protoc exch [Crossref]

43. Judenhofer MS1, Wehrl HF, Newport DF, Catana C, Siegel SB, et al. (2008) Simultaneous PET-MRI: a new approach for functional and morphological imaging. Nat Med 14: 459-465. [Crossref] 
Webb E (2016) Imaging in animal models

44. Pichler B, Wehrl H, Kolb A, Judenhofer M (2008) Positron Emission Tomography/ Magnetic Resonance Imaging: The Next Generation of Multimodality Imaging? Seminars Nucl Med 38: 199-208. [Crossref]

45. Solomon M, Nothdruft R, Akers W, Edwards W, Liang K, et al. (2013) Multimodal
Fluorescence-Mediated Tomography and SPECT/CT for Small-Animal Imaging. $J$ Nucl Med 54: 639-646. [Crossref]

46. Condeelis J, Weissleder R (2010) In vivo imaging in cancer. Cold Spring Harb Perspect Biol 2: a003848. [Crossref]

Copyright: (C) 2016 Webb E. This is an open-access article distributed under the terms of the Creative Commons Attribution License, which permits unrestricted use, distribution, and reproduction in any medium, provided the original author and source are credited. 